\title{
Síntesis y caracterización de nanoparticulas de CdS obtenidas por microondas
}

\author{
S. MARTíNEZ, T. SERRANO, I. GÓMEZ, A. HERNÁNDEZ \\ Facultad de Ciencias Químicas, UANL. \\ Av. Pedro de Alba s/n, Cd. Universitaria, San Nicolás de los Garza, N.L. México
}

\begin{abstract}
En este trabajo se presentan los resultados de la síntesis de nanoparticulas semiconductoras de CdS por medio de microondas. Se usó Tioacetamida (TAA) y $\mathrm{CdCl}_{2}$ para la obtención de las nanoparticulas, se controló térmicamente con microondas a una potencia de $1000 \mathrm{~W}$, un tiempo de 60 segundos bajo el ajuste de diferentes valores de $\mathrm{pH}: 8,9$ y 10 . Se caracterizaron los nanocompuestos mediante espectroscopia UV-Vis con reflectancia difusa, Análisis textural a partir de las isotermas de adsorción con $\mathrm{N}_{2}$ usando el método BET, Microscopia de Fuerza Atómica y Difracción de Rayos X en polvos. Los resultados del análisis textural demuestran una distribución de tamaño de poros estrecha, así como área superficial de hasta $106.4 \mathrm{~m}^{2} / \mathrm{g}$. Los difractogramos indican la obtención de CdS con tamaños de cristal nanométricos, el cual fue confirmado por el análisis por espectroscopia de UV-Vis así como por fuerza atómica. Las curvas de absorbancia de UV-Vis indicaron una disminución de tamaño de nanopartículas en función del pH utilizado, así como un incremento en la energía de banda prohibida en relación inversa al tamaño de éstas, cuyos valores van desde 2.08 hasta $2.29 \mathrm{eV}$.
\end{abstract}

Palabras clave: microondas, semiconductor, CdS, nanoparticulas.

Synthesis and characterization of CdS NP'S by microwaves

This paper present the results of the synthesis and characterization of semiconductor nanoparticles of CdS by microwaves. Thioacetamide (TAA) and $\mathrm{CdCl}_{2}$ were the initial materials. The synthesis was carried out under controlled potency of $1000 \mathrm{~W}$, with 60 seconds on or off, as well as the $\mathrm{pH}$ was regulated to different values: 8,9 and 10 . The nanocompounds were analyzed by UV-Vis spectroscopy, Textural Analysis from adsorption isoterms with $\mathrm{N}_{2}$ by BET method, Atomic Force Microscopy and X-Ray Diffraction. Textural analysis shows the size of pore distribution narrow, as well as superficial area of $106.4 \mathrm{~m}^{2} / \mathrm{g}$. AFM micrographies shown particle nanometrics, the results of X-Ray Difraction analysis probing the same. Spectras of UV-Vis shown that nanoparticle is less according the $\mathrm{pH}$ used in the synthesis, affecting the band gap energy.

Keywords: microwaves, semiconductor, CdS, nanoparticles.

\section{INTRODUCCIÓN}

En la época actual, la necesidad de nuevos materiales que satisfagan las peticiones que los avances tecnológicos requieren, ha encauzado a la investigación de recientes campos del conocimiento a virar el rumbo del esfuerzo científico a una nueva era en el estudio de la ciencia de los materiales, aportando como resultado, el desarrollo de una naciente pero interesante rama del conocimiento, la nanociencia.

Las propiedades de los sólidos cristalinos son generalmente clasificadas sin tomar como referencia su tamaño ya que éste generalmente solo tiene influencia cuando se trabaja con sistemas de tamaños menores a $10 \mathrm{~nm}$ (1). El desarrollo de materiales y la síntesis de cristales inorgánicos de una manera controlable ha sido la meta de muchas investigaciones en la actualidad, cuyo principal interés es el control del tamaño (2-7).

En los últimos años se ha revelado un especial interés en semiconductores a escala nanométrica, en especial en los sulfuros de los metales de transición, semiconductores que adquieren aplicación como sensores, filtros ópticos, celdas solares, sistemas fotocatalíticos, entre otras $(6,8)$. Los nanocristales de los semiconductores están siendo estudiados extensivamente debido a sus propiedades ópticas, las cuales son altamente dependientes del tamaño y morfología del cristal, ya que las variaciones en las características fundamentales que se muestran en la fase de transición a la conductividad eléctrica, pueden inducirse controlando el tamaño del cristal (9-11).

Usando estos nuevos conocimientos, se pretende mejorar los materiales ya existentes para un uso más efectivo, tal es el caso de lo que se intenta efectuar con la síntesis de nanoparticulas semiconductoras de CdS, ya que éste tiene propiedades ópticas que no se observan normalmente en otra clase de materiales semiconductores. Dichas propiedades se modifican particularmente al disminuir el tamaño de partícula, además, éstas son diferenciadas cuando la estructura cristalina de la nanopartícula cambia $(12,13)$.

El material semiconductor CdS, puede presentar dos estructuras cristalinas, una de ellas es una estructura cúbica (tipo blenda de cinc) y la otra es una estructura cristalina hexagonal (tipo wurtzita). Los semiconductores representan una clase de bloques construidos a nanoescala y que han sido utilizados para construir estructuras electrónicas incluyendo los diodos emisores de luz, fotodetectores, láser altamente 
eficientes y sistemas fotocatalíticos. El buen funcionamiento de un semiconductor esta ligado a propiedades importantes como su cristalinidad y el tamaño de las nanopartículas. Una de las preguntas más interesantes es cómo las propiedades moleculares son muy remarcadas cuando el tamaño de las nanoparticulas de los semiconductores decrece (14-17).

En las transformaciones del estado sólido no se puede olvidar la influencia que ejercen las interacciones entre los átomos en la superficie del nanocristal, ya que éstas pueden alterar la energía relativa de la superficie de alguna de las dos fases, por lo que el tamaño debe ser controlado por el así llamado terminación o truncamiento de crecimiento. El acoplamiento de compuestos orgánicos en la superficie del nanocompuesto ha sido utilizado para detener el proceso de crecimiento $(10,11,18)$, y el acoplamiento de compuestos inorgánicos previene la morfología (19).

Ahora bien, en los años 40 se comienza a utilizar el microondas como una efectiva herramienta de calentamiento, principalmente en la industria alimenticia. Fue hasta la década de los años 80 que esta técnica fue introducida a estudios meramente científicos y su aplicación se extendió al calificarla como una efectiva herramienta de trabajo en la síntesis de materiales a altas temperaturas sin el peligro de la exposición a ellas, además, mejoró considerablemente los tiempos de proceso.

Esta técnica fue utilizada principalmente en la síntesis de compuestos orgánicos, investigaciones de las cuales se encuentran numerosos trabajos publicados (20-23). La aplicación de esta técnica en áreas inorgánicas ha avanzado más lentamente debido a que el calentamiento con microondas puede provocar una ligera variación en las propiedades mecánicas del compuesto sintetizado (21). Por otra parte, se sabe que la eficacia de esta metodología se debe principalmente a las interacciones del momento bipolar de las moléculas presentes con la frecuencia de radiación, por lo tanto se puede deducir que el agua es un excelente solvente para las reacciones que involucren microondas por su alto momento bipolar. Esta ruta de síntesis se ha utilizado para producir componentes inorgánicos desde 1986 (24-27). Comparado con otros métodos convencionales la síntesis por microondas tiene como ventaja que es una reacción que se realiza en poco tiempo y produce pequeñas partículas con una baja distribución de tamaño y alta pureza.

El objetivo planteado en este trabajo es el de sintetizar nanoparticulas de sulfuro de cadmio (CdS) bajo un campo de microondas de $2.45 \mathrm{GHz}$ provocando así un truncamiento en el crecimiento de las mismas.

\section{PROCEDIMIENTO EXPERIMENTAL}

\subsection{Reactivos}

Se utilizó $\mathrm{CdCl}_{2}$ como precursor del catión [Spectrum Quality Products, Inc; Assay 95.0\%], Tioacetamida $\left(\mathrm{CH}_{3} \mathrm{CSNH}_{2}\right)$ como precursor del anión [MERCK Gehalt 99.0\%], KOH, Metanol y Agua Destilada.

\subsection{Síntesis}

Se preparó una solución con $0.1125 \mathrm{~g}$ de Tioacetamida y una segunda solución con $0.1374 \mathrm{~g}$ de $\mathrm{CdCl}_{2}$. Una tercera solución se preparó para controlar el $\mathrm{pH}$ de la reacción, la cual se realizó con $1.50 \mathrm{~g}$ de $\mathrm{KOH}$ a un volumen de
40mL. Posteriormente se mezclaron las tres soluciones y se sometieron a un tratamiento térmico en un horno de microondas de $2.45 \mathrm{GHz}$ a $1000 \mathrm{~W}$ de potencia máxima durante 60 segundos. Se controló el pH en tres niveles: 8, 9 y 10.

Los sólidos obtenidos se pasaron a tubos de ensayo, se agregó metanol como agente de lavado y posteriormente se trataron por ultrasonido en agua durante 10 minutos para eliminar los subproductos, centrifugando y dejando secar las muestras a temperatura ambiente por un lapso de 24 horas en la oscuridad.

\subsection{Caracterización}

Los sólidos obtenidos se caracterizaron mediante análisis de espectroscopia de UV-Vis con reflectancia difusa, Análisis Textural, Microscopia de Fuerza Atómica, Análisis Elemental y Difracción de Rayos X. El análisis por UV-Vis se realizó en un aparato Perkin Elmer de UV-Vis Lambda 12 con esfera de integración Labsphere. Las pruebas texturales se realizaron en un aparato Quantachrome Autosorb Automated Gas Sorption. El análisis elemental se realizó mediante una sonda de EDX acoplada a un microscopio electrónico de barrido LEICA S440. La difracción de rayos X, se llevó a cabo en un Difractómetro Siemens D5000 utilizando radiación $\mathrm{Cu}$ $\mathrm{K} \alpha(\lambda=1.5418 \mathrm{~A})$. La intensidad fue medida en el rango entre $10^{\circ}$ y $85^{\circ}$ con un paso de $0.05^{\circ}$.

\section{RESUTADOS Y DISCUSIÓN}

Después de realizado el procedimiento experimental se recuperaron sólidos de color anaranjado en diferentes intensidades de tono, estando éste en función del $\mathrm{pH}$ utilizado.

Dos espectros de absorción del análisis por UV-Vis del nanocompuesto $\mathrm{CdS}$ obtenido por microondas a valores de $\mathrm{pH}: 8,9$ y 10 son mostrados en la figura 1, los espectros fueron barridos de 300 a $700 \mathrm{~nm}$.

Dichos espectros mostraron pruebas del efecto de confinamiento cuántico que indica la formación de partículas de tamaño nanométrico. Trabajos teóricos muestran que el umbral de absorción de la longitud de onda del espectro de absorción del UV-Vis proporciona una estimación razonable del tamaño de partícula (7), en función de la posición y espaciamiento de estos. Los cambios del espectro de absorción del sulfuro de cadmio cambian con el tamaño de partícula, esto demuestra que variando el $\mathrm{pH}$ de la reacción se pueden obtener diferentes tamaños de partículas y que esta disminución del tamaño afecta las propiedades físicas del CdS. Las partículas más grandes que son aproximadamente de $10 \mathrm{~nm}$ comienzan a absorber cerca de $600 \mathrm{~nm}$. Cuando el tamaño del nanocristal disminuye el compuesto empieza a absorber a longitudes de onda más cortas (520nm), tal es el caso del espectro de absorción de la muestra que se obtuvo controlando la reacción a $\mathrm{pH} 8$, lo cual indica que al disminuir el valor de $\mathrm{pH}$ en la reacción se obtienen menores tamaños de partícula. En el espectro de absorción de la muestra que se obtuvo cuando la reacción se controló a un valor de $\mathrm{pH} 10$ se puede observar claramente que se tienen partículas mayores.

Con la reducción del tamaño de partícula, el ancho de banda del semiconductor se hace mayor y hay un fenómeno observable de traslación en los espectros de emisión de la Figura 1, esto es, el movimiento de la curva de absorción 


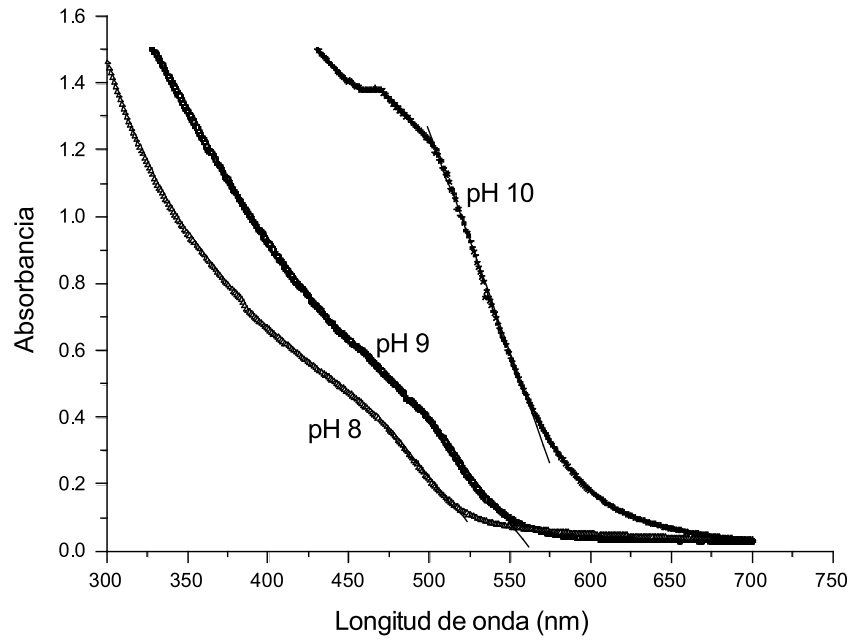

Fig. 1- Espectros UV-Vis de CdS sintetizado a diferentes valores de $\mathrm{pH}$.

hacia longitudes de onda menores. A esto se le llama el efecto del tamaño cuántico de la partícula del semiconductor $(2,3,5,28)$. Los valores de la banda de energía prohibida del sólido fueron calculados a partir de la ecuación $\alpha(\mathrm{h} v)=\mathrm{A}(\mathrm{h} v-$ $\left.\mathrm{E}_{\mathrm{g}}\right)^{\mathrm{m} / 2}$, donde $\alpha$ es el coeficiente de absorción, hv es la energía del fotón y $\mathrm{m}=1$ para una transición directa entre las bandas (de valencia y banda de conducción). A partir de estos espectros de UV-vis, la $\mathrm{E}_{\mathrm{g}}$ fue calculada por extrapolación de una línea recta a partir de la curva de absorción hacia el eje de la abscisa. Cuando $\alpha$ es igual a cero, entonces $E_{g}$ $=\mathrm{h} v$. La longitud de onda en nanómetros correspondiente a dicha extrapolación se convierte en unidades de energía en electrón volts (eV). Para el compuesto obtenido en los diferentes niveles de $\mathrm{pH}$ la extrapolación corresponde a los valores mostrados en la tabla 1. En estos resultados se puede apreciar un aumento en la energía de banda prohibida en función de la disminución de tamaño de partícula, sin embargo cabe mencionar que el valor de energía de banda prohibida reportada para este compuesto es de $2.42 \mathrm{eV}$ (29), por lo que los valores encontrados demuestran el efecto del tamaño del nanocristal en la estructura de bandas de este compuesto, lo cual resulta de la disminución en el deslizamiento molecular del orbital con disminución en las dimensiones atómicas del arreglo, por lo que la longitud de onda de absorción presenta un corrimiento hacia el azul (30).

Para calcular el área superficial del producto se usó el método de Brunauer-Emmett-Teller, (BET), que se basa en la adsorción física de un gas en la superficie sólida. Generalmente se utiliza nitrógeno como adsorbato y se determina la cantidad de gas adsorbido en el equilibrio a su punto de ebullición normal $\left(-195.8^{\circ} \mathrm{C}\right)$ en un intervalo de presiones inferiores a una atmósfera. Los datos obtenidos son los volúmenes de gas adsorbido a una serie de presiones en la cámara de adsorción. La figura 2 muestra las isotermas de adsorción de nitrógeno por el compuesto obtenido a los diferentes valores de $\mathrm{pH}$ utilizados.

Los valores obtenidos del área superficial del CdS sintetizado, se muestran en la tabla 1 . En estos resultados puede apreciarse que se obtuvo una mayor área superficial a un menor $\mathrm{pH}$.

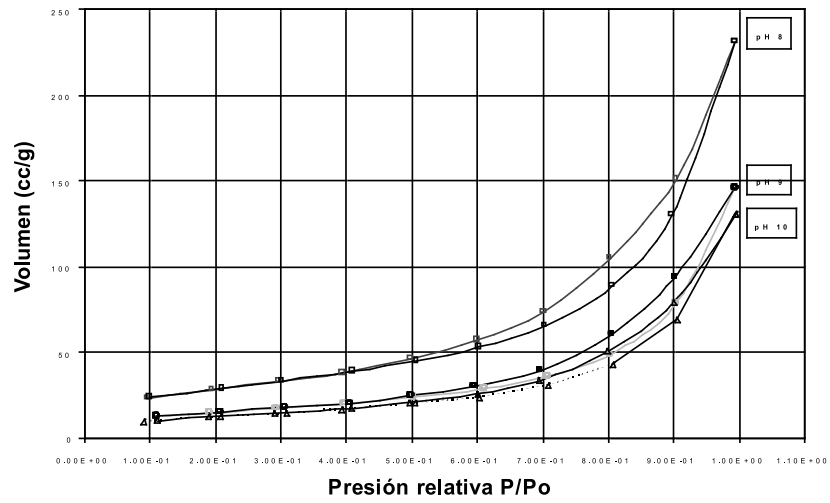

Fig. 2- Isotermas de Adsorción para el nanocompuesto CdS.

Elárea superficial de las nanoparticulas esta íntimamente relacionada con el tamaño de éstas, por lo que se observa que existe una relación entre los resultados del área superficial con los datos obtenidos del análisis por UV-Vis. A mayor área superficial se obtiene un tamaño de partícula mucho

TABLA I. ÁREA SUPERFICIAL Y ENERGÍA DE BANDA PROHIBIDA DEL CdS SINTETIZADO

\begin{tabular}{|c|c|c|}
\hline $\mathrm{pH}$ & $\begin{array}{c}\text { Área Superficial } \\
\left(\mathrm{m}^{2} / \mathrm{g}\right)\end{array}$ & $\begin{array}{c}\mathrm{Eg} \\
(\mathrm{eV})\end{array}$ \\
\hline 8 & 106.4 & 2.29 \\
\hline 9 & 56.7 & 2.20 \\
\hline 10 & 47.6 & 2.08 \\
\hline
\end{tabular}

menor y una menor absorción de longitud de onda a medida que el tamaño de la partícula decrece, en estos resultados se puede observar que en el experimento a $\mathrm{pH} 8$ se obtiene un área superficial muy grande con tan solo disminuir una unidad el valor del $\mathrm{pH}$ de la síntesis.

En la figura 3 se muestra una imagen de microscopía de fuerza atómica de una de las muestras del CdS sintetizado a un $\mathrm{pH}$ de 10, la imagen se obtuvo de un análisis del CdS sintetizado, el cual fue depositado vía precipitación de

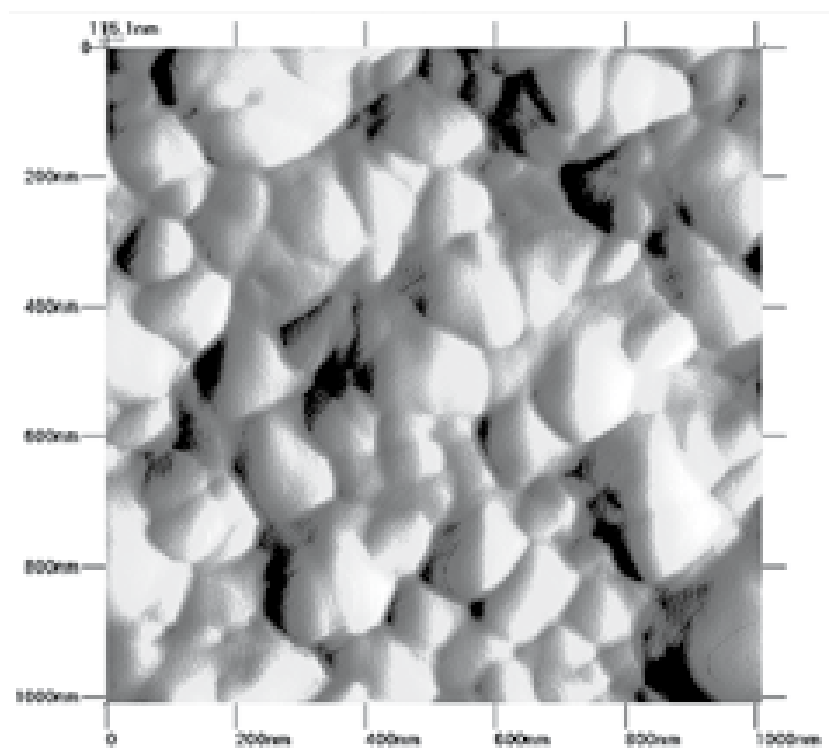

Fig. 3- Imagen de Microscopía de Fuerza Atómica del CdS sintetizado. 


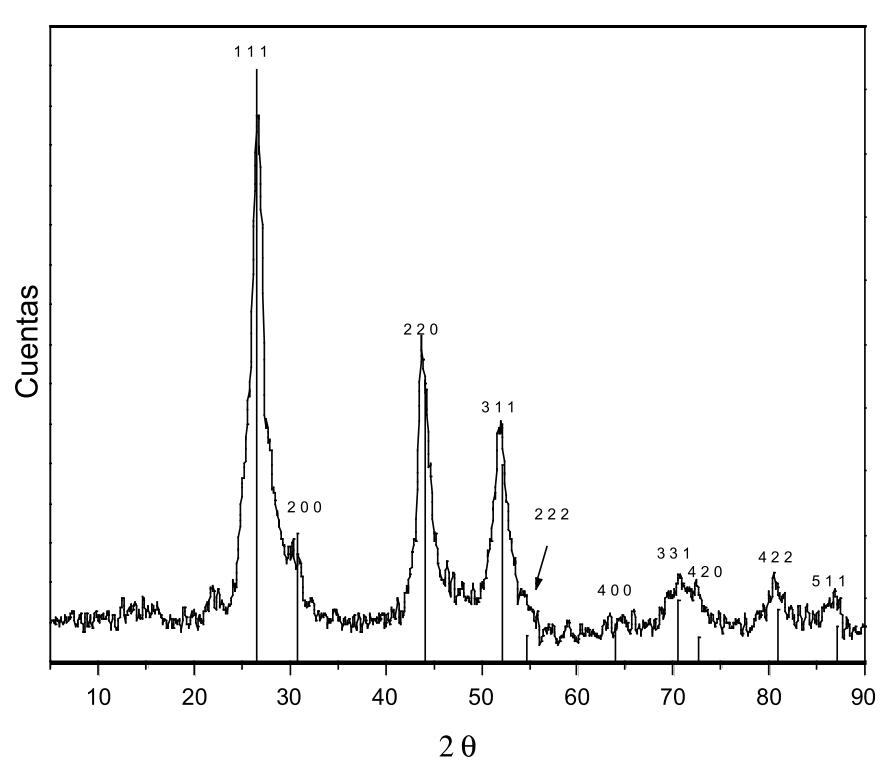

Fig. 4- Difractograma del compuesto CdS sintetizado.

una suspensión preparada. En esta micrografía se pueden observar partículas esféricas de CdS con tamaños que van de 50 a $100 \mathrm{~nm}$, así como aglomerados los cuales se formaron debido a la preparación de la muestra para su análisis por esta técnica, ya que su alta área superficial y el tiempo implicado en ello favoreció la coalescencia de partículas.

El análisis elemental se realizó mediante EDX, en la tabla

TABLA II. ANÁLISIS ELEMENTAL DEL CdS SINTETIZADO

\begin{tabular}{|c|c|}
\hline Elemento & $\%$ en Peso \\
\hline $\mathrm{O}$ & 8.2 \\
\hline $\mathrm{Si}$ & 0.8 \\
\hline $\mathrm{S}$ & 20.6 \\
\hline $\mathrm{K}$ & 2.0 \\
\hline $\mathrm{Cd}$ & 68.4 \\
\hline Total & 100.0 \\
\hline
\end{tabular}

2 se muestran los resultados, en donde se puede apreciar además del contenido de Azufre y Cadmio, la presencia de Potasio, Oxígeno y Silicio, los primeros dos provienen del compuesto utilizado para controlar el $\mathrm{pH}(\mathrm{KOH})$, mientras que la pequeña cantidad de Silicio proviene del desgaste del vaso de precipitados utilizado para la síntesis en microondas.

El análisis mineralógico del compuesto obtenido se realizó por difracción de rayos $\mathrm{X}$ en polvos. En la figura 5 se presenta el espectro de difracción obtenido, en donde se aprecia que este coincide con las reflexiones reportadas para el compuesto CdS con estructura cúbica (blenda de zinc) la cual fue determinada mediante análisis del monocristal (800019 JCPDS), esto permite asegurar que el nanocompuesto sintetizado es CdS.

\section{CONCLUSIONES}

Se sintetizaron nanopartículas de CdS mediante el truncamiento de crecimiento por efecto de microondas. Existe una relación directa entre el $\mathrm{pH}$ de síntesis y el tamaño de nanopartícula de CdS, pero en relación inversa con el área superficial y energía de banda prohibida, obteniéndose menor tamaño de partícula a un $\mathrm{pH}$ de 8 , con un área superficial de hasta $106.4 \mathrm{~m}^{2} / \mathrm{g}$ y energía de banda prohibida de $2.29 \mathrm{eV}$.

\section{AGRADECIMIENTOS}

A los laboratorios de Vía Húmeda y Sol-Gel, así como también al laboratorio de Cerámica Tradicional ambos de la Facultad de Ciencias Químicas. A la dirección de la Facultad de Ciencias Químicas y al Programa PAICYT por el apoyo económico para el desarrollo de esta línea de investigación.

\section{BIBLIOGRAFÍA}

1. A.P. Alivisatos. "Perspectives on the Physical Chemistry of Semiconductor Nanocrystals". J. Phys. Chem. 1996, 100, 13226-13239.

2. Arnim Henglein. "Small-Particle Research: Physicochemical Properties of Extremely Small Colloidal Metal and Semiconductor Particles". Chem. Rev. 1989, 89, 1861-1873.

3. Cheng Tao; Suping Zheng; Helmuth Möhwald; Junbai Li. "CdS Crystal Growth of Lamellar Morphology within Templates of Polyelectrolyte/ Surfactant Complex". Langmuir. 2003, 19, 9039-9042.

4. Ch. Barglik-Chory; Ch. Remenyi; H. Strohm; G. Müller."Adjustment of the Band Gap Energies of Biostabilized CdS Nanoparticles by Application of Statistical Design of Experiments". J. Phys. Chem. B. 2004, 108, 7637-7640.

5. Manoj E. Wankhede; Santosh K. Haram. "Synthesis and Characterization of Cd-DMSO Complex Capped CdS Nanoparticles". Chem. Mater. 2003, 15, 1296-1301.

6. Vijay T. John; Gary L. McPherson. “Morphology of CdS Nanocrystals Synthesized in a Mixed Surfactant System". Nano Lett. 2002, Vol. 2, No. 4, 263-268.

7. Angela Agostiano. "Synthesis and Characterization of CdS Nanoclusters in a Quaternary Microemulsion: the Role of the Cosurfactant". J. Phys. Chem. B. 2000, 104, 8391-8397.

8. Nicholas A. Kotov."CdS Nanoparticles Modified to Chalcogen Sites: New Supramolecular Complexes, Butterfly Bridging, and Related Optical Effects". J. Am. Chem. Soc. 2002, 124, 3980-3992.

9. Chien M. Wai. "Water-in- $\mathrm{CO}_{2}$ Microemulsions as Nanoreactors for Synthesizing CdS and ZnS Nanoparticles in Supercritical $\mathrm{CO}_{2}^{\prime \prime}$. Nano Lett. 2002, Vol. 2, No. 7, 721-724.

10. A. Paul Alivisatos. "Band Gap Variation of Size- and Shape-Controlled Colloidal CdSe Quantum Rods". Nano Lett. 2001, Vol. 1, No. 7, 349-351.

11. Takayuki Hirai."Stabilization of CdS Nanoparticles Immobilized on ThiolModified Polystyrene Particles by Encapsulation with Polythiourethane". J. Phys. Chem. B. 2001, 105, 9711-9714.

12. H.Weller. “CdS Nanoclusters: Synthesis, Characterization, Size Dependent Oscillator Strength, Temperature Shift of the Excitonic Transition Energy, and Reversible Absorbance Shift". J. Phys. Chem. 1994, 98, 7665-7673.

13. Lian Gao. "Synthesis and Characterization of CdS Nanorods via Hydrothermal Microemulsion". Langmuir. 2003, 19, 208-210.

14. H. S. Zhou. “Coated Semiconductor Nanoparticles: The CdS/PbS System's Synthesis and Properties". J. Phys. Chem. 1993, 97, 895-901.

15. M. P. Pileni. "Oil in Water Micellar Solution Used To Synthesize CdS Particles: Structural Study and Photoelectron Transfer Reaction". Langmuir. 1994, 10, 4446-4450.

16. Mostafa A. El-Sayed."Picosecond Electronic Relaxation in CdS/HgS/CdS Quantum Dot Quantum Well Semiconductor Particles". J. Phys. Chem. 1996, 100, 6381-6384

17. Mingyuan Gao. "Enhancement Effect of Illumination on the Photoluminescence of Water-Soluble CdTe Nanocrystals: Toward Highly Fluorescent CdTe/CdS Core-Shell Structure". Chem. Matter. 2004, 16, 3853-3859.

18. Guoshong Cao. "Nanostructures and nanomaterials". Imperial College Press. 2004.

19. R. Pozas, M.P. Morales, C.J. Serna, M. Ocaña. “Acicular iron Nanoparticles protected against sintering with aluminium oxide" Bol. Soc. Esp. Ceram. V., 43 [4] 796-800 (2004). 
20. C. Ludlow-Palafoz, H. Chase. "Microwave-induced pyrolysis of plastic wastes". Ind. Eng. Chem. Res. 40200122 4749-4756.

21. G.M.B. Parkes, P.A. Barnes, G. Bond and E.L. Charsley. “Qualitative and quantitative aspects of microwave thermal analysis". Thermochimica Acta 3562000 1-2 85-96.

22. A.C. Metaxas. "Industrial Microwave Heating". Peter peregrinus Ltd, 1983.

23. P. Benito, F.M. Labajar, V. Rives "Effect of microwave radiation on the cristallinity of layered materials" Bol. Soc. Esp. Ceram. V., 43 [1] 56-58 (2004).

24. Laurel M. Sheppard. "Manufacturing Ceramics with Microwaves: The potential for Economical Production". Ceram. Bull. 67198810 1656-61.

25. Palaith David and Silberglitt Richard. "Microwaves Joining of Ceramics". Ceram. Bull. 68198991600 .
26. Idalia Gómez, Maryangel Hernández, Juan Aguilar, Moisés Hinojosa. "Comparative Study of Microwave and Conventional processing of $\mathrm{MgAl}_{2} \mathrm{O}_{4}$-based Materials". J. Int. Cer. 3020046 893-900.

27. G. Vargas, J.C. Pérez, J. Méndez, M. Méndez, P. Pena. “Espumado por microondas de mezclas de silicato de sodio-ortofosfato cálcico dibásico". Bol. Soc. Esp. Ceram. V., 41 [5] 481-486 (2002).

28. M. P. Pileni. "Synthesis of Cadmium Sulfide in Situ in Cadmium Bis(ethyl-2-hexyl) Sulfosuccinate Reverse Micelle: Polydispersity and Photochemical Reaction". Langmuir. 1992, 8, 1049-1053.

29. J. Richard Christman. "Fundamentals of Solid State Physics". John Wiley \& Sons. 1988.

30. Nathan S. Lewis and Mary L. Rosenbluth. "Theory of Semiconductor Materials", Peter peregrinus Ltd, 1983.

Recibido: $\quad$ 13.10.05

Aceptado: 06.03 .06 